\title{
Effects of Synthetic Copolymer and Algae-Exopolysaccharides for the Sedimentation and Dehydration of Organic Waste
}

\author{
Nana Yamada-Kato ${ }^{1,2}$, Akihisa Okuda ${ }^{2}$, Minoru Fukaya ${ }^{3}$, Teruhiro Takabe ${ }^{1,4}$, Tetsuko Takabe $^{1 *}$ \\ ${ }^{1}$ Research Institute, Meijo University, Nagoya, Japan \\ ${ }^{2}$ Act Co., Ltd., Konan, Japan \\ ${ }^{3}$ Faculty of Science \& Technology, Meijo University, Nagoya, Japan \\ ${ }^{4}$ Graduate School of Environmental and Human Sciences, Meijo University, Nagoya, Japan
}

\begin{abstract}
"Corresponding author: Tetsuko Takabe, Research Institute of Meijo University, Tenpaku-ku, Nagoya, Aichi 468-8502, Japan. Tel: +81528382277; Fax: +81528321545; E-mail: tptakabe@meijo-u.ac.jp

Citation: Yamada-Kato N, Okuda A, Fukaya M, Takabe T, Takabe T (2017) Effects of Synthetic Copolymer and Algae-Exopolysaccharides for the Sedimentation and Dehydration of Organic Waste. J Agr Agri Aspect: JAAA-119.
\end{abstract}

Received Date: 08 May, 2017; Accepted Date: 30 June, 2017; Published Date: 08 July, 2017

\begin{abstract}
Summary
Significant amounts of organic waste are produced in agriculture and water treatments. Many flocculants are used to decrease the volume of sludge. But, the water content in the sludge cake is still high $(>80 \%)$ when it is treated with flocculants followed by mechanical dewatering. These sludge cakes are incinerated using oil or landfilled, which causes expenses and environmental effects. Here, we compared the effects of synthetic-polymer (styrene/acrylic acid copolymer) and extracellular polysaccharide from a halotolerant cyanobacterium Aphanothece halophytica (ApEPS) on the sedimentation and dehydration of organic waste. The synthetic copolymer enhanced the flocculation of clay particles whereas the flocculation of clay particles by exopolysaccharide was only effective at low $\mathrm{pH}(<2.5)$. When the sludge cake was incubated with the synthetic copolymer $(5 \%)$ in a draft at $30^{\circ} \mathrm{C}$, the water content decreased less than $20 \%$ at earlier time than that in the sludge cake without use of the synthetic copolymer (control). These findings indicate the usefulness of synthetic copolymer for dehydration whereas further improvement is required for biological polymers.
\end{abstract}

Keywords: Dehydration; Extracellular Polysaccharide; Flocculation; Synthetic Copolymer

\section{Abbreviations:}

Ap : Aphanothece halophytica

BSA : $\quad$ Bovine Serum Albumin

EPS : $\quad$ Extracellular Polysaccharide

\section{Introduction}

Many flocculants are used in the treatment of organic waste to decrease the volume of sludge. Flocs generated by various coagulation mechanisms exhibit different size, strength and structure, these elements have been found to control sludge dewaterability (Chen et al. 2001; Li et al. 2006) [1,2]. Metal-ion coagulants (e.g. poly-aluminium chloride and polyferric sulphate) and organic polymers are often used (Matilainen et al. 2010) [3]. The water content in sludge cake can be reduced to approximately $80 \%$ when it is treated with these coagulants followed by mechanical dewatering (Chen et al. 2001; Murakami et al. 2009) [1,4]. The resulting sludge cake is used for landfill or incinerated together with oil. The landfill with sludge cake has been recognized to cause various environmental issues. The incineration together with oil has run up costs and causes the high $\mathrm{CO}_{2}$ emission. If the water content in the sludge cake can be reduced less than $20 \%$, it can be used as a fuel for thermal power plant (Japanese Industrial Standards, JIS). In this study, we examined to develop a technique to decrease the water content in sludge cake without use of high energy.

Two possibilities were tested. One is the use of synthetic copolymer, emulsion of styrene/acrylic acid copolymer. The other 


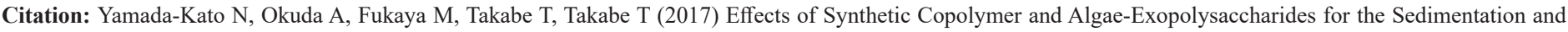
Dehydration of Organic Waste. J Agr Agri Aspect: JAAA-119.

is extracellular polysaccharide from a halotolerant cyanobacterium Aphanothece halophytica (ApEPS). Previously, Chen et al (2010) [5] reported that A. halophytica often forms algal blooms in salterns in summer of China and releases large amounts of polysaccharide. ApEPS was shown to have a unique property of claydispersing activity which was stable during the $\mathrm{pH}$ range 3.0-10.0 (Li et al. 2001; Chen et al. 2010) [5,6]. Our A. halophytica was isolated from Dead Sea and can grow at high external $\mathrm{NaCl}$ concentrations up to $3 \mathrm{M}$ and at alkaline pH 11 (Takabe et al. 1988; Laloknam et al. 2006) $[7,8]$. The genetic identity of both $A$. halophytica is unknown, but the similarity is probably high.

Development of dehydration systems is important for the management of organic waste to use as a renewable energy sources and to decrease $\mathrm{CO}_{2}$ emission. In the present study, we compared the effects of synthetic copolymer and ApEPS on the sedimentation and dehydration of sludge, and a possible application of copolymer for dehydration was discussed.

\section{Materials and Methods}

\section{Growth Condition}

A. halophytica was grown photoautotrophically in BG11 liquid medium plus $18 \mathrm{mM} \mathrm{NaNO}_{3}$ and Turk Island salt solution containing $0.5 \mathrm{M} \mathrm{NaCl}$ at $30^{\circ} \mathrm{C}$ with shaking $(120 \mathrm{rpm})$ under continuous fluorescent white light $\left(70 \mu \mathrm{E} \mathrm{m}^{-2} \cdot \mathrm{s}^{-1}\right)$ (Takabe et al. 1988; Waditee et al. 2001) [7,9]. Growth was monitored by measuring the optical density at $730 \mathrm{~nm}$ (OD730) with a Shimadzu UV-160A spectrophotometer.

\section{Preparation of $A$. halophytica Extracellular Polymeric Substance (ApEPS) for Sedimentation and Dehydration}

Cultures were grown for 28 days and then centrifuged at $1,680 \times \mathrm{g}$ for 10 minutes. The pellet was discarded, and the supernatant was filtered through $0.22 \mu \mathrm{m}$ filters. The ApEPS was precipitated by the addition of 4 volumes of cold ethanol and then centrifuged at $4,500 \times \mathrm{g}$ for $15 \mathrm{~min}$ at $4^{\circ} \mathrm{C}$. The pellets were dissolved in distilled water, transferred into dialysis tubes of which cut-off molecular weight is $14,000 \mathrm{Da}$, and dialyzed against distilled water at $4{ }^{\circ} \mathrm{C}$ for $24 \mathrm{~h}$. The dialysate was concentrated using Amicon Ultra (Millipore, MA, USA) and washed three times with distilled water.

\section{Kaolin Sedimentation}

Kaolin clays were added into a graduated cylinder which contained distilled water or 3\% acetic acid supplemented. Then, the emulsion copolymer or ApEPS wad added. The final volume was adjusted to $100 \mathrm{~mL}$ and the clay concentration was $6 \%$. The suspensions were mixed thoroughly by vigorous shaking 15 times and allowed to stand at room temperature for $10 \mathrm{~min}$. Then, the suspensions were once again mixed and allowed to stand at room temperature.

\section{Cake Dehydration}

Organic-sludge-cake which was dehydrated by mechanical dewatering (Imuraya, Tsu, Japan) was used. Its water content was about $85 \%$. The sludge cake $(9.5 \mathrm{~g})$ was mixed with $0.5 \mathrm{~g}$ copolymer or ApEPS. The well mixed cakes $(0.5 \pm 0.02 \mathrm{~g})$ were rounded up to a ball. The balls were put on the aluminum blocks. Then the balls of cakes were kept at $30^{\circ} \mathrm{C}$ with blowing $(0.5 \mathrm{~m} / \mathrm{s})$ air at relative humidity less than $40 \%$. Dehydration was monitored by measuring the water content with a Shimadzu MOC63u moisture meter.

\section{Measurement of Polysaccharide and Protein Content}

The preparation of ApEPS and extracellular proteins were performed as described previously (Chen et al. 2010) [5]. The $A$. halophytica cultures were centrifuged at $1,680 \mathrm{x}$ g for $10 \mathrm{~min}$ at $20^{\circ} \mathrm{C}$. The supernatant was filtrated with $0.22 \mu \mathrm{m}$ filters. The filtrate was applied to a column of Amicon Ultra (Millipore, MA, USA) with cutoff molecular weight of 3,000 Da, and washed three times with distilled water. Polysaccharide content in the filtrate was measured by the phenol-sulfuric acid method (Dubois et al. 1956) [10] using glucose as standard, and the protein content was determined by Bradford (1976) [11] method using BSA as standard.

\section{SDS-PAGE and Staining with Stains-All}

SDS-PAGE was carried out according to the standard protocol (Waditee et al. 2005) [12]. Proteins were visualized by staining with Coomassie Brilliant Blue (CBB). The protein staining with Stains-All was carried out as described (Yuasa and Maeshima 2001) [13]. To detect highly acidic proteins, polyacrylamide gels were stained with the metachromatic cationic carbocyanine dye Stains-all (Sigma Aldrich, Tokyo; Campbell et al. 1983) [14]. After electrophoresis, the gel was fixed with $25 \%(\mathrm{v} / \mathrm{v})$ isopropyl alcohol and $30 \mathrm{mM}$ Tris. Then the gel was stained in the dark for $24 \mathrm{~h}$ with $0.0025 \%$ (wt/v) Stains-all, 25\% (v/v) isopropyl alcohol, $7.5 \%(\mathrm{v} / \mathrm{v})$ formamide and $30 \mathrm{mM}$ Tris, $\mathrm{pH} 8.8$.

\section{Alcian Blue Stain}

The polysaccharide was stained with Alcian blue (Stockwell and Scott 1965; Tamaru et al. 2005; Porsch et al. 2012) [15-17]. Cells were washed with distilled water, placed in 3\% acetic acid for $30 \mathrm{~min}$, and then placed in Alcian blue reagent ( $\mathrm{pH} 2.5$ ) containing 1\% Alcian blue 8GX solution (Siguma-Aldorich, Tokyo, Japan) in 3\% acetic acid to stain acid mucopolysaccharides. The samples were washed with distilled water to remove excess dye. The SDS-PAGE gel was stained for $2 \mathrm{~h}$ with $0.125 \%$ Alcian blue, $40 \%$ ethanol and 5\% acetic acid and then de-stained overnight 
with $40 \%$ ethanol and $5 \%$ acetic acid.

\section{Results and Discussion}

\section{Chemical Copolymer Enhanced the Sedimentation of Clay Particle Kaolin whereas Exopolysaccharide was only Effective at low pH}

Clay particle kaolin has often been used for the test of coagulation activity. We examined the effects of synthetic copolymer and EPS for the coagulation of kaolin. Figure 1A shows that the interfacial sedimentation of the $6 \%$ kaolin suspension in water (closed circle) was slow. The sedimentation interface was slowly decreased at a constant rate from $16 \mathrm{~cm}$ to $13 \mathrm{~cm}$ during $7 \mathrm{~min}$ incubation. However, if the $1 \%$ synthetic copolymer was included with the $6 \%$ kaolin suspension, the interface was rapidly decreased during $3 \mathrm{~min}$ and almost reached to a plateau at the height of $6 \mathrm{~cm}$ after 7 min (Figure 1A, open square). When ApEPS was included with the $6 \%$ kaolin suspension, the decrease of interfacial sedimentation was the same to that of water (Figure 1A, open triangle). Li et al (2010) reported that ApEPS has clay-dispersing activity which is stable at the $\mathrm{pH}$ range 3-10 and inhibit flocculation. Our data are consistent with the results of $\mathrm{Li}$ et al (2010). However, we observed that if ApEPS was included with the $6 \%$ kaolin suspension in $3 \%$ acetic acid $(\mathrm{pH} 2.5)$, the interface sedimentation was enhanced (Figure 1A, closed triangle). These data indicate that the synthetic copolymer has strong sedimentation activity for kaolin, and the clay-sedimentation activity of ApEPS was only observed at $\mathrm{pH}<2.5$. ApEPS has clay-dispersing activity at neutral $\mathrm{pH}$ which is unique among various kinds of EPS (Li et al. 2010). Microscopy observation showed that the size of kaolin floc incubated with ApEPS in distilled water was very small (Figure 1D). Its size was slightly smaller than that in distilled water (Figure 1B), which is compatible with the clay-dispersing activity of ApEPS at neutral $\mathrm{pH}$. Larger flocs were observed when ApEPS was included at acidic $\mathrm{pH} 2.5$ (Figure 1E). The synthetic copolymer produced the largest flocs (Figure 1C). The results of floc size (Figures 1B-E) are consistent with the order of sedimentation activity.
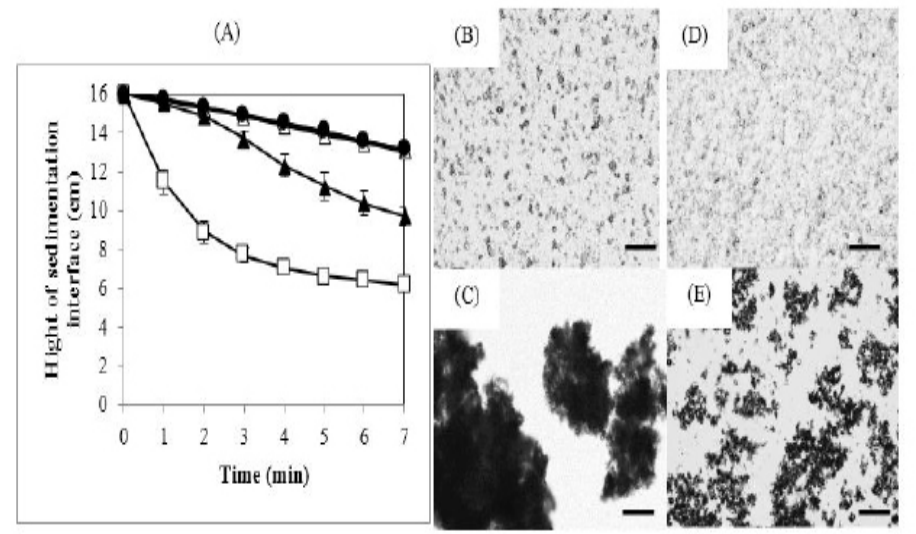

Figure 1: Flocculation of kaoline aqueous suspension. (A) Sedimentation curves for selected flocculants. Each aqueous suspension contained $6 \%$ kaolin in distilled water (closed circle), $1 \%$ synthetic copolymer (open square) or $3.24 \mathrm{mg} / \mathrm{L} \mathrm{ApEPS}$ in distilled water (open triangle) or 3\% acetic acid (closed triangle). Each value shows the average of three independent measurements. Resulting flocks, $1 \mathrm{~cm}$ below the sedimentation interface, were observed by light microscopy (B)-(E). The flocculation solution contained distilled water (B), synthetic copolymer in distilled water (C), ApEPS in distilled water, and ApEPS in 3\% acetic acid (E). Scale bars represent $100 \mu \mathrm{m}$.

\section{Chemical Copolymer Accelerated the Dehydration of Sludge Cake}

Next, we tested whether the water content in the sludge cake can be decreased to less than $20 \%$ without the use of high energy. Before treatment, the water content in our sludge cake was about $85 \%$ as shown in Figure 2. When the sludge cake was put on the plate of which temperature was $30^{\circ} \mathrm{C}$, the water content decreased with the increase of incubation time as shown in Figure 2A (open circle). After $5 \mathrm{~h}$, the water content was slightly higher than $20 \%$. If the synthetic copolymer was included in the sludge cake (3-5 $\%$ ), the water content in the sludge cake (open square) decreased faster than the control (Figure 2A). These facts indicate that the water content can be decreased lower than $20 \%$ after $5 \mathrm{~h}$ incuba- 


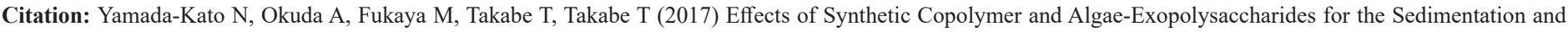
Dehydration of Organic Waste. J Agr Agri Aspect: JAAA-119.

tion together with copolymer. By contrast, when ApEPS was included in the sludge cake, the decrease of water content (closed triangle) was the same as that of the control (Figure 2B). These data indicate that synthetic copolymer is a useful dehydration-reagent for sludge cake.
(A)

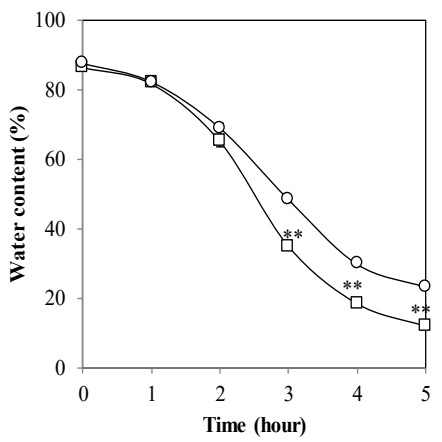

(B)

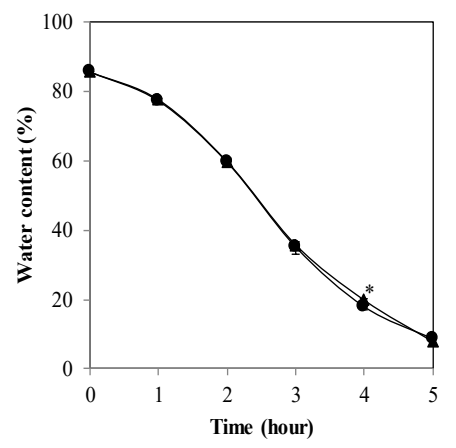

Figure 2: Time-course-changes of water contents in dehydrated cake. (A) Effects of synthetic copolymer. The cakes contained distilled water (control, opened circle) or $5 \%(\mathrm{v} / \mathrm{w})$ synthetic copolymer (opened square). (B) Effects of ApEPS. The cakes contained 3\% (v/w) acetic acid (control, closed circle)) or $16.2 \mu \mathrm{g} / \mathrm{g}$ ApEPS in 3\% (v/w) acetic acid (closed triangle). The data represent the mean \pm standard deviation of (A) nine and (B) three experiments. ${ }^{* *} \mathrm{P}<0.01$.

\section{The ApEPS Contained Acidic Proteins that were Stained with Stain-All}

The above data showed that ApEPS was not suitable for the dehydration of sludge cake. Since the clay-dispersing activity of ApEPS is unique among various kinds of EPS (Li et al. 2001, Chen et al. 2010) [5,6], we further investigated the properties of extracellular macromolecules of A. halophytica cells. Figures 3A and 3B showed that the accumulation of extracellular polysaccharides and proteins of $A$. halophytica increased with increasing the growth time when the growth medium contains $0.5 \mathrm{M} \mathrm{NaCl}$. At high salinity conditions $(2.5 \mathrm{M} \mathrm{NaCl})$, a similar pattern was observed although their increase was lower than that at lower salinity $(0.5 \mathrm{M}$ $\mathrm{NaCl}$ ) (data not shown). Figure 3C showed that the extracellular polysaccharides could be stained with Alcian blue (Stockwell and Scott 1965) [15] which indicates that ApEPS is acid mucopolysaccharide (Li et al. 2001) [6]. Figure 3D showed that several extracellular proteins can be stained with Coomassie Brilliant Blue (CBB) (left). Among them, a $40 \mathrm{kDa}$ band was stained with Alcian blue (middle), suggesting this protein is a glycoprotein. Light panel of Figure 3D showed that almost all extracellular proteins were stained with Stains-All, a metachromatic cationic carbocyanine dye. Stain-All has been used for identification of calcium binding proteins (Campbell et al. 1983) [14].
(A)

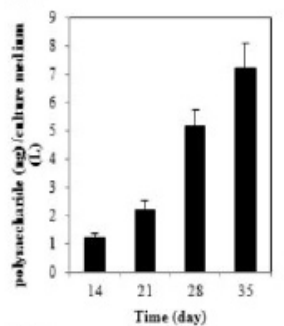

(B)

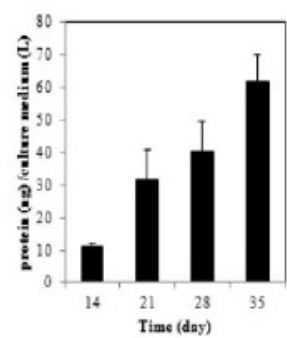

(C)

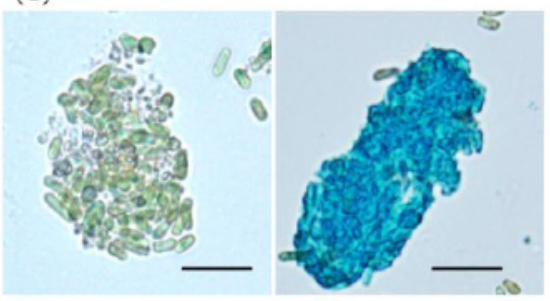

(D)

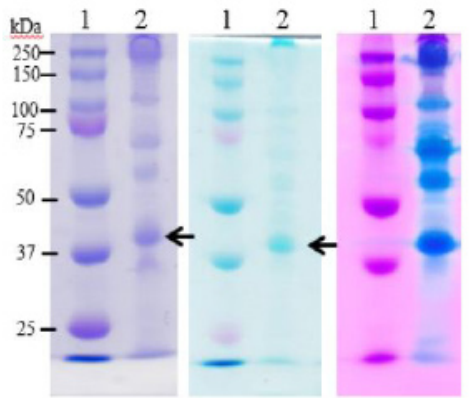

Figure 3: Growth and accumulation of extracellular polysaccharides and proteins. (A) The growth of A. halophytica cells. (B) The content of extracellular polysaccharides. Each value shows the average of three independent measurements. (C) Polysaccharide stain. The cells were grown for 20 days and then photographs were taken by light microscopy. Left panel, Control; Right panel, Alcian Blue stain. Scale bars represent $20 \mu \mathrm{m}$. (D) Left panel, Coomassie brilliant blue staining; Middle panel, Alcian Blue staining; Right panel, Stains-All staining. Extracellular polysaccharides and proteins were prepared by ammonium sulfate precipitation.

\section{Effects of Light and $\mathrm{CaCl}_{2}$ on the Flocculation of $\mathrm{A}$. halo- phytica cells}

The concentration of $\mathrm{Ca}^{2+}$ in Dead Sea is high $(0.43 \mathrm{M})$, and Figure 3D suggests that $A$. halophytica cells excrete many putative $\mathrm{Ca}^{2+}$ binding proteins. Therefore, we examined the effects of $\mathrm{Ca}^{2+}$ on the flocculation of $A$. halophytica cells. Our standard growth medium of $A$. halophytica cells contained $10 \mathrm{mM} \mathrm{CaCl}_{2}$. Figure 4A showed that the growth rate of $A$. halophytica cells was almost constant up to $200 \mathrm{mM} \mathrm{CaCl}_{2}$. Figure 4B showed the absorbance of growth medium at $730 \mathrm{~nm}$ after $24 \mathrm{~h}$-incubation under light and dark conditions. The cotton plug cap was used for the incubation under light whereas the sealed cap with parafilm was used for the dark-incubation. Higher absorbance at $730 \mathrm{~nm}$ was observed after the incubation under light (Figure 4B, open circle), compared with the incubation under anaerobic dark (closed circle). The results indicate that the dark-anaerobic-incubation enhanced the flocculation of A. halophytica cells. The reason for this effect is not clear. The recent reports showed that the cyanobacterium Synechocystis sp. PCC 6803 excretes organic acids and hydrogen under dark-anaerobic conditions (Osanai et al. 2015; Ueda et al. 2016) [18,19]. It might be possible that more anionic compounds under dark incubation enhanced the flocculation of A. halophytica cells. Further studies are required to clary the mechanisms. 
Citation: Yamada-Kato N, Okuda A, Fukaya M, Takabe T, Takabe T (2017) Effects of Synthetic Copolymer and Algae-Exopolysaccharides for the Sedimentation and Dehydration of Organic Waste. J Agr Agri Aspect: JAAA-119.

(A)

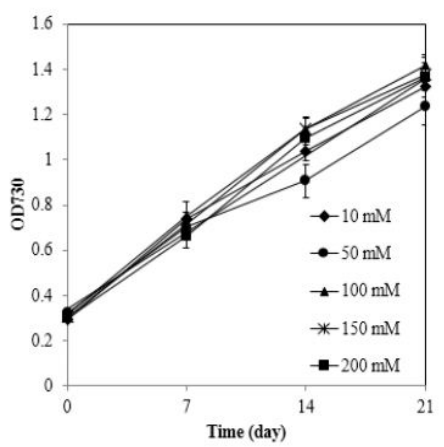

(B)

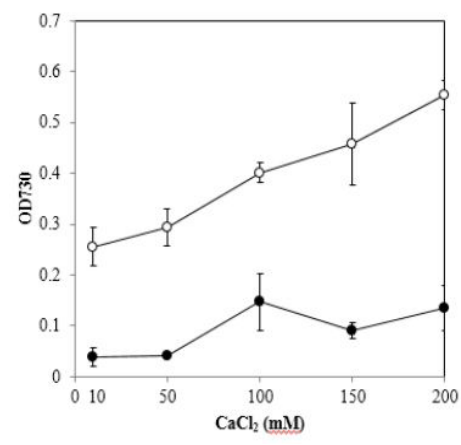

Figure 4: Effect of $\mathrm{CaCl}_{2}$ on the growth and flocculation activity of $A$. halophytica cells. (A) Growth curve of $A$. halophytica under various Ca$\mathrm{Cl}_{2}$ concentrations. (B) Flocculation of $A$. halophytica cells under various $\mathrm{CaCl}_{2}$ concentrations. Cultures were grown for 21 days under various $\mathrm{CaCl}_{2}$ concentrations. Then, cells were divided into $2 \mathrm{~mL}$ tubes. After the tubes were incubated at $30^{\circ} \mathrm{C}$ for $24 \mathrm{~h}$ under light aerobic conditions (opened circle) or dark anaerobic conditions (closed circle), the turbidity of the upper $250 \mu \mathrm{L}$ samples was measured at $730 \mathrm{~nm}$ (OD730). Each value shows the average of three independent measurements.

\section{Conclusion}

Alternative method to decrease the water content in organic waste was presented in this article. It was shown that a synthetic copolymer is a useful reagent to decrease the water content less than $20 \%$ whereas many improvements are required for biopolymers from A. halophytica cells. To realize these compounds in commercial basis, large scale demonstration test would be required using rotational dryers.

\section{Acknowledgments}

This work was supported by Strategic foundational technology advancement support project (The small and medium enterprise agency in Japan) and the International Research Center for Natural Environmental Science in Meijo University.

\section{References}

1. Chen Y, Yang H, Gu G (2001) Effect of acid and surfactant treatment on activated sludge dewatering and settling. Water Res 35: 2615-2620.

2. Li T, Zhu Z, Wang D, Yao C, Tang H (2006) Characterization of floc size, strength and structure under various coagulation mechanisms. Powder Technol 168: 104-110.

3. Matilainen A, Vepsäläinen M, Sillanpää M (2010) Natural organic matter removal by coagulation during drinking water treatment: a review. Adv Colloid Interfac 159: 189-197.

4. Murakami T, Suzuki Y, Nagasawa H, Yamamoto T, Koseki T, et al. (2009) Combustion characteristics of sewage sludge in an incineration plant for energy recovery. Fuel Process Technol 90: 778-783.
5. Chen L, Men X, Ma M, Li P, Jiao Q, et al. (2010) Polysaccharide release by Aphanothece halophytica inhibits Cyanobacteria/Clay Flocculation. J Phycol 46: 417-423.

6. Li P, Liu Z, Xu R (2001) Chemical characterisation of the released polysaccharide from the cyanobacterium Aphanothece halophytica GR02. J Appl Phycol 13: 71-77.

7. Takabe T, Incharoensakdi A, Arakawa K, Yokota S (1988) $\mathrm{CO}_{2}$ fixation rate and RuBisCO content increase in the halotolerant cyanobacterium, Aphanothece halophytica, grown in high salinities. Plant Physiol 88: $1120-1124$

8. Laloknam S, Tanaka K, Buaboocha T, Waditee R, Incharoensakdi A, et al. (2006) Halotolerant cyanobacterium Aphanothece halophytica contains a betaine transporter active at alkaline $\mathrm{pH}$ and high salinity. Appl Environ Microbiol 72: 6018-6026.

9. Waditee R, Hibino T, Tanaka Y, Nakamura T, Incharoensakdi A, et al. (2001) Halotolerant Cyanobacterium Aphanothece halophytica Contains an $\mathrm{Na}^{+} / \mathrm{H}^{+}$Antiporter, Homologous to Eukaryotic Ones, with Novel Ion Specificity Affected by C-terminal Tail. J Biol Chem 276: 3693136938.

10. Dubois M, Gilles KA, Hamilton JK, Rebers PA, Smith F (1956) Colorimetric method of determination of sugars and related substances. Anal Chem 28: 350-356.

11. Bradford MM (1976) A rapid and sensitive method for the quantitation of microgram quantities of protein utilizing the principle of protein-dye binding. Anal Biochem 72: 248-254.

12. Waditee R, Bhuiyan MNH, Rai V, Aoki K, Tanaka Y, et al. (2005) Genes for direct methylation of glycine provide high levels of glycinebetaine and abiotic-stress tolerance in Synechococcus and Arabidopsis. Proc Natl Acad Sci USA 102: 1318-1323.

13. Yuasa K, Maeshima M (2001) Organ specificity of a vacuolar $\mathrm{Ca}^{2+}$ binding protein $\mathrm{RvCaB}$ in radish and its expression under $\mathrm{Ca}^{2+}$-deficient conditions. Plant Mol Biol 47: 633-640.

14. Campbell KP, Mac Lennan DH, Jorgensen AO (1983) Staining of the $\mathrm{Ca}^{2+}$-binding proteins, calsequestrin, calmodulin, troponin $\mathrm{C}$, and S-100, with the cationic carbocyanine dye 'Stains-All'. J Biol Chem 258: $11267-11273$

15. Stockwell RA and Scott JE (1965) Observations on the acid glycosaminoglycan (mucopolysaccharide) content of the matrix of aging cartilage. Ann Rheum Dis 24: 341-350.

16. Tamaru Y, Takani Y, Yoshida T, Sakamoto T (2005) Crucial role of extracellular polysaccharides in desiccation and freezing tolerance in the terrestrial cyanobacterium Nostoc commune. Appl Environ Microbiol 71: 7327-7333.

17. Porsch EA, Kehl-Fie TE, Geme JWS (2012) Modulation of Kingella kingae adherence to human epithelial cells by type IV pili, capsule, and a novel trimeric autotransporter. MBio 3: e00372-12.

18. Osanai T, Shirai T, lijima H, Nakaya Y, Okamoto M, et al. (2015) Genetic manipulation of a metabolic enzyme and a transcriptional regulator increasing succinate excretion from unicellular cyanobacterium. Front Microbiol 6: 1064.

19. Ueda S, Kawamura Y, lijima H, Nakajima M, Shirai T, et al. (2016) Anionic metabolite biosynthesis enhanced by potassium under dark, anaerobic conditions in cyanobacteria. Sci Rep 6: 32354. 